\title{
Reproducción, distribución y abundancia del pez Pseudupeneus grandisquamis (Perciformes: Mullidae), en el Golfo de Tehuantepec, México
}

\author{
Eduardo Ramos-Santiago, José Martín Ramírez-Gutiérrez, Ricardo Mendoza-Rodríguez \& \\ Margarito Tapia-García \\ Departamento de Hidrobiología. D.C.B.S. Universidad Autónoma Metropolitana-Iztapalapa. Apartado Postal 55-535, \\ México D.F. Fax 58044738; mtg@xanum.uam.mx
}

Recibido 08-VII-2003. C Corregido 07-VII-2005. Aceptado 26-VI-2006.

\begin{abstract}
Reproduction, distribution and abundance of the fish Pseudupeneus grandisquamis (Perciformes: Mullidae), in the Gulf of Tehuantepec, Mexico. As result of its biological and ecological strategies, Pseudupeneus grandisquamis is a dominant species in the demersal community of the Gulf of Tehuantepec, Mexico. Our main objective was to understand these strategies with respect to distribution, abundance and reproduction. We analyzed 5175 individuals representing partial collections from five oceanographic expeditions between 1989 and 1990. It is a typical demersal marine species, with a wide distribution on the continental shelf. The highest abundance of $P$. grandisquamis occurs in March and November, around the $40 \mathrm{~m}$ isobath, facing the Superior-Inferior lake systems and Mar Muerto Lagoon. Reproduction occurred during all of the months studied, particularly from August to October, corresponding to the rainy season, when the salinity and temperature is lower. The presence of juveniles, principally in November and March, suggests a long period of recruitment; they are distributed mainly in the Superior-Inferior Lagoons, which serves as a nursery area where they remain until they are adults. The total female to male sex ratio was nearly 1:1 throughout the year. The maximum total length was $213 \mathrm{~mm}$ and the size at first maturity was $138 \mathrm{~mm}$ TL. The high abundance and reproduction occur when the gulf has a high level of ecological production, in accordance with the dynamics of the system, where the influence of coastal lagoons is important. Protection strategies for the area above the continental shelf of the Gulf of Tehuantepec are recommended for the estuary processes and for the reproduction and rearing of a large number of species, including P. grandisquamis. Rev. Biol. Trop. 54 (4): 1103-1112. Epub 2006 Dec. 15.
\end{abstract}

Key words: Pseudupeneus grandisquamis, reproduction, demersal, Tehuantepec, ecology, dominance, lagoons.

El Golfo de Tehuantepec, en la costa sur del Pacífico mexicano, es una zona de intensa actividad pesquera e industrial (petróleo), que lo coloca en una situación prioritaria en cuanto a la necesidad de evaluación ecológica de sus recursos. En esta región, la actividad pesquera está orientada principalmente al recurso camarón y por lo tanto a las especies demersales asociadas o fauna de acompañamiento, y esta actividad representa el cuarto lugar en cuanto a la producción nacional de camarón que es la divisa pesquera más importante en México.

Los recursos demersales de alta diversidad del Golfo de Tehuantepec, característicos de áreas tropicales, están constituidos por más de 170 especies de peces, que en el ámbito comunitario tienen patrones de distribución y abundancia que responden a la dinámica ecológica de la región, entre las que sobresalen 20 especies dominantes (Tapia García 1998). Las especies dominantes, por su importancia biológica y ecológica, tienen un papel clave en la estructura y función de las comunidades, lo que ha determinado que las investigaciones sobre recursos demersales costeros de alta diversidad se orienten a la información que aportan estas especies como un reflejo de la dinámica de toda la comunidad. Esta estrategia de investigación 
se menciona en los trabajos de Pauly (1982) y Yáñez-Arancibia y Sánchez-Gil (1988), donde se considera como punto importante el conocimiento de las diferentes etapas biológicas y de dinámica poblacional de las especies clave para el entendimiento de la estructura y función de las comunidades demersales.

La información que existe sobre las poblaciones de peces demersales dominantes en el Golfo de Tehuantepec ha abierto una ruta de investigación con grandes perspectivas, donde se han detectado necesidades de estudios sobre la estructura y función de las poblaciones y su relación con la dinámica del ecosistema. Entre las 20 especies dominantes informadas, se encuentra Pseudupeneus grandisquamis por su alta abundancia e incidencia en las capturas demersales. El estudio de esta especie es de gran relevancia ante su importancia biológica y ecológica, y su potencial económico como una especie susceptible de ser explotada.

$P$. grandisquamis se caracteriza por ser típicamente marina, ya que no hay informes que indiquen su distribución dentro de sistemas estuarinos. En el Pacífico mexicano, existen diversos trabajos que informan a esta especie como un componente característico de la ictiofauna demersal, y Allen y Robertson (1998) mencionan su presencia en fondos lodosos, arenosos, rocosos y en praderas de pastos asociadas a arrecifes coralinos. Sin embargo, no existen antecedentes biológicos y de dinámica poblacional a pesar de su importancia biológica, ecológica y como recurso pesquero potencial. Por lo anterior, el objetivo del presente trabajo es caracterizar el patrón espacial y temporal de la distribución y abundancia de $P$. grandisquamis con relación a su reproducción y reclutamiento.

\section{MATERIALES Y MÉTODOS}

La plataforma continental del Golfo de Tehuantepec se encuentra localizada en el sureste del Pacífico mexicano, entre las Bahías de Huatulco, Oaxaca, y el Río Suchiate, Chiapas (Fig. 1). Se analizaron las recolectas obtenidas

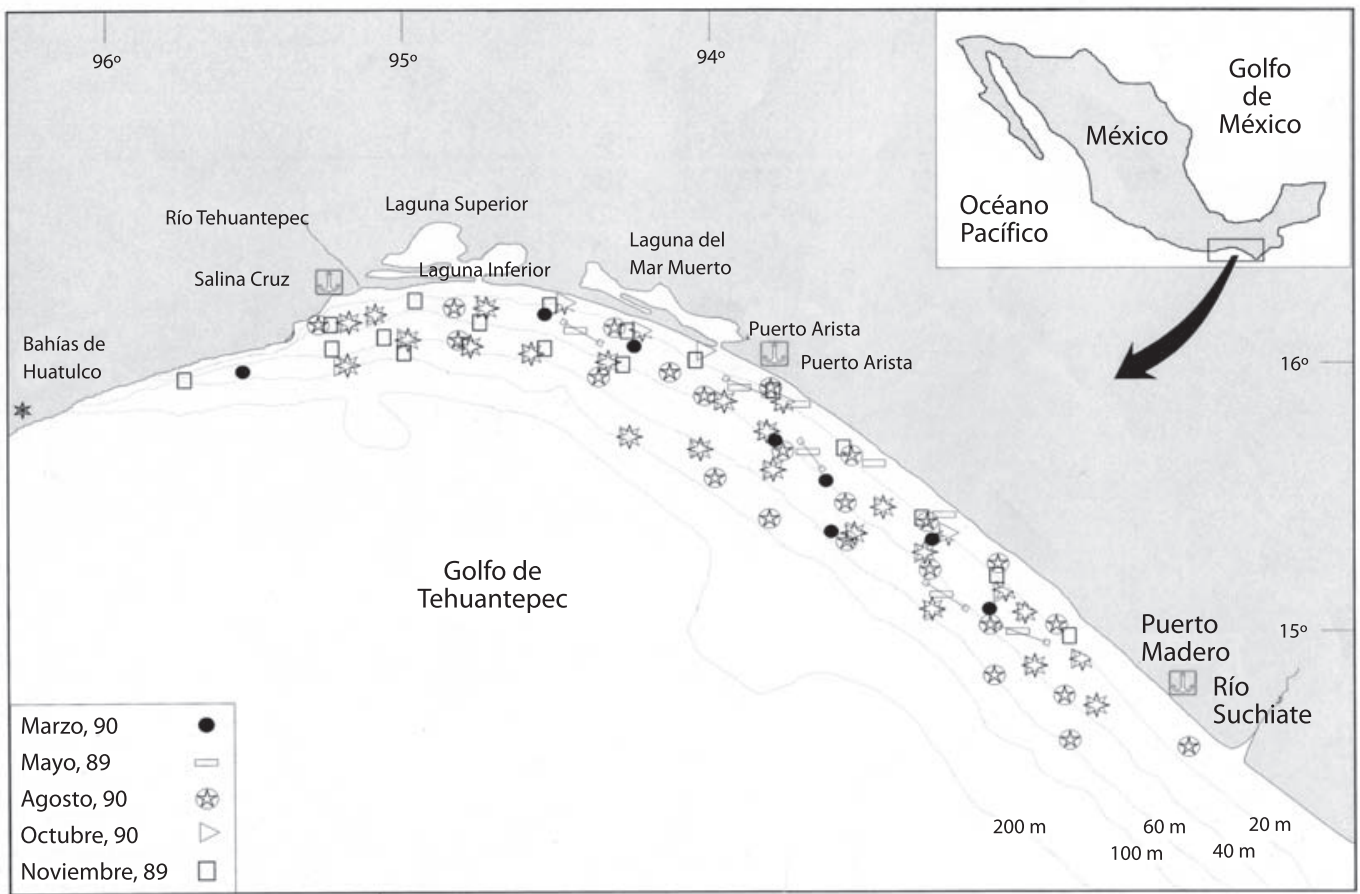

Fig. 1. Área de estudio. Las estaciones de recolecta se muestran en relación a cada crucero oceanográfico.

Fig. 1. Study area. The collect stations are showed in relation to oceanographic cruises. 
en cinco cruceros oceanográficos en mayo y noviembre de 1989, y marzo, agosto y octubre de 1990. Las recolectas de mayo, agosto y noviembre se realizaron en el buque oceanográfico "El Puma", y la de marzo y octubre en el buque motor "Don Nachito". Se realizaron un total de 86 puntos de muestreo entre los 15 y $80 \mathrm{~m}$ de profundidad (Fig. 1). Se utilizó una red de arrastre camaronera con un tamaño de boca de 9 (B/M Don Nachito) y 12 m (B/O El Puma), luz de malla de $4.45 \mathrm{~cm}$ a una velocidad promedio de 3 nudos durante $30 \mathrm{~min}$. El área de arrastre, la captura de los peces y el procesamiento de los datos se hizo con base a los criterios discutidos por Stevenson (1982) y Sánchez-Gil y Yáñez-Arancibia (1985).

Cada individuo se sexó y se le midió la longitud total (mm), el peso total (g) (peso húmedo), peso sin vísceras, peso de gónadas, y se determinaron los parámetros poblacionales de densidad (individuos $/ \mathrm{m}^{2}$ ), biomasa $\left(\mathrm{g} / \mathrm{m}^{2}\right)$, longitud y peso promedio. El índice visceral y el gonadosomático fueron determinados por sexo, estación y mes de recolecta. Los datos corresponden a dos años diferentes, y fueron ordenados mensualmente para asumir un periodo anual.

Madurez gonádica: Para conocer el estado de madurez gonádica se utilizó la clave presentada en Laevastu (1971). Estadio I = inmaduro (jóvenes), sexualmente indeterminados; II inmaduros (jóvenes), sexualmente determinados; III en maduración; IV maduros; V en reproducción; VI desovados y VII en descanso.

Talla de primera madurez: Se determinó por medio del punto intermedio, entre la longitud de la hembra inmadura más grande (II) y la hembra madura más pequeña (III) (TapiaGarcía et al. 1995).

Índice gonadosomático (GSI): Fue evaluado de acuerdo a Cailliet et al. (1986):

$$
\mathrm{GSI}=\mathrm{B} / \mathrm{W}
$$

donde W es el peso húmedo del organismo, y B es el peso húmedo de ambas gónadas.
Índice visceral (VI): Se evaluó con la siguiente ecuación:

$$
\mathrm{VI}=\mathrm{V} / \mathrm{W}
$$

donde $\mathrm{W}$ es el peso del individuo y $\mathrm{V}$ es el peso de las vísceras.

\section{RESULTADOS}

Distribución y abundancia: Se capturaron un total de 5175 individuos de $P$. grandisquamis, con un peso total de $201.9 \mathrm{~kg}$, y un intervalo de tallas de 67 a $213 \mathrm{~mm}$ de longitud total (Cuadro 1). Se distribuye en profundidades menores a los $60 \mathrm{~m}$, sobre sedimentos arenosos y lodosos. La mayor densidad $(>0.00154$ ind $\left./ \mathrm{m}^{2}\right)$ y biomasa $\left(>0.0605 \mathrm{gr} / \mathrm{m}^{2}\right)$ se presenta alrededor de la isobata de los $40 \mathrm{~m}$, frente a la Laguna del Mar Muerto, principalmente en marzo, agosto y noviembre (Figs. 2A, B). La densidad indica una alta concentración de individuos durante los meses de marzo y noviembre $\left(\mathrm{x}=0.0082 \mathrm{y} \mathrm{x}=0.0015 \mathrm{ind} / \mathrm{m}^{2}\right.$ respectivamente), y en mayo la concentración mas baja ( $\mathrm{x}=$ $0.0002 \mathrm{ind} / \mathrm{m}^{2}$ ). La biomasa tiene un comportamiento similar a la densidad, el valor más alto se presenta en marzo y agosto $(\bar{x}=0.0959)$ y el mínimo en mayo $\left(\bar{x}=0.0033 \mathrm{gr} / \mathrm{m}^{2}\right)$ (Fig. 3B). La talla y peso promedio muestran un

CUADRO 1

Abundancia total y longitud de $\mathrm{P}$. grandisquamis por crucero oceanográfico

TABLE 1

Total abundance and length of $\mathrm{P}$. grandisquamis by oceanographic cruise

\begin{tabular}{lccc}
\multicolumn{1}{c}{ Crucero } & $\begin{array}{c}\text { Número } \\
\text { (Ind) }\end{array}$ & $\begin{array}{c}\text { Peso } \\
(\mathrm{g})\end{array}$ & $\begin{array}{c}\text { Long. Total } \\
(\mathrm{mm})\end{array}$ \\
Marzo, 1990 & 281 & 7098.2 & $94-190$ \\
Mayo, 1989 & 14 & 392.9 & $128-168$ \\
Agosto, 1990 & 278 & 8645.5 & $117-213$ \\
Octubre, 1991 & 223 & 4639.5 & $134-173$ \\
Noviembre, 1989 & 97 & 1862.6 & $67-186$ \\
Total de captura & 931 & 22638.7 & $67-213$
\end{tabular}




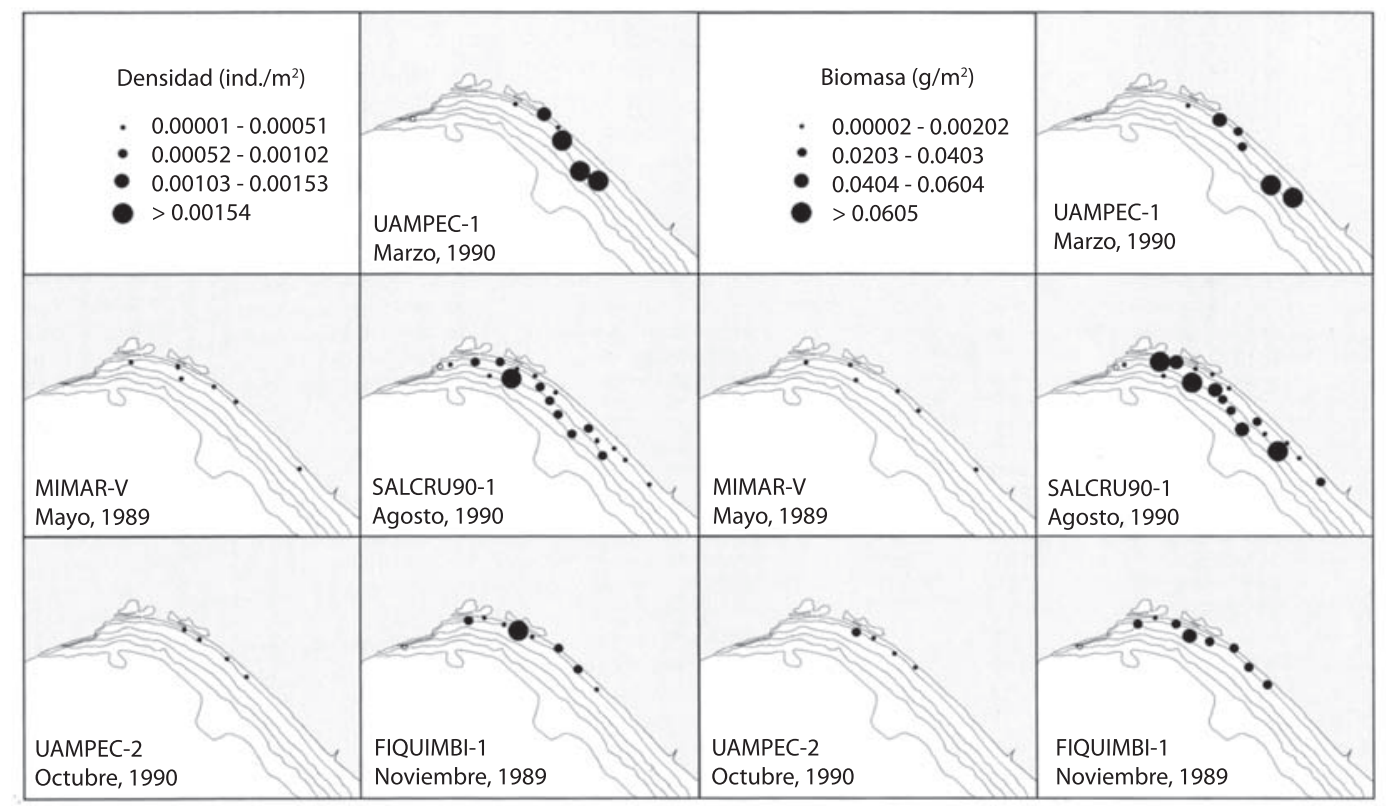

A

B

Fig. 2. A. Distribución de la densidad. B. Distribución de la biomasa en el Golfo de Tehuantepec. Los circulos vacíos indican que la especie no fue encontrada en esas estaciones.

Fig. 2. A. Distribution of density. B. Distribution of biomass in the Gulf of Tehuantepec. Empty circles indicate that the species was not found in those stations.
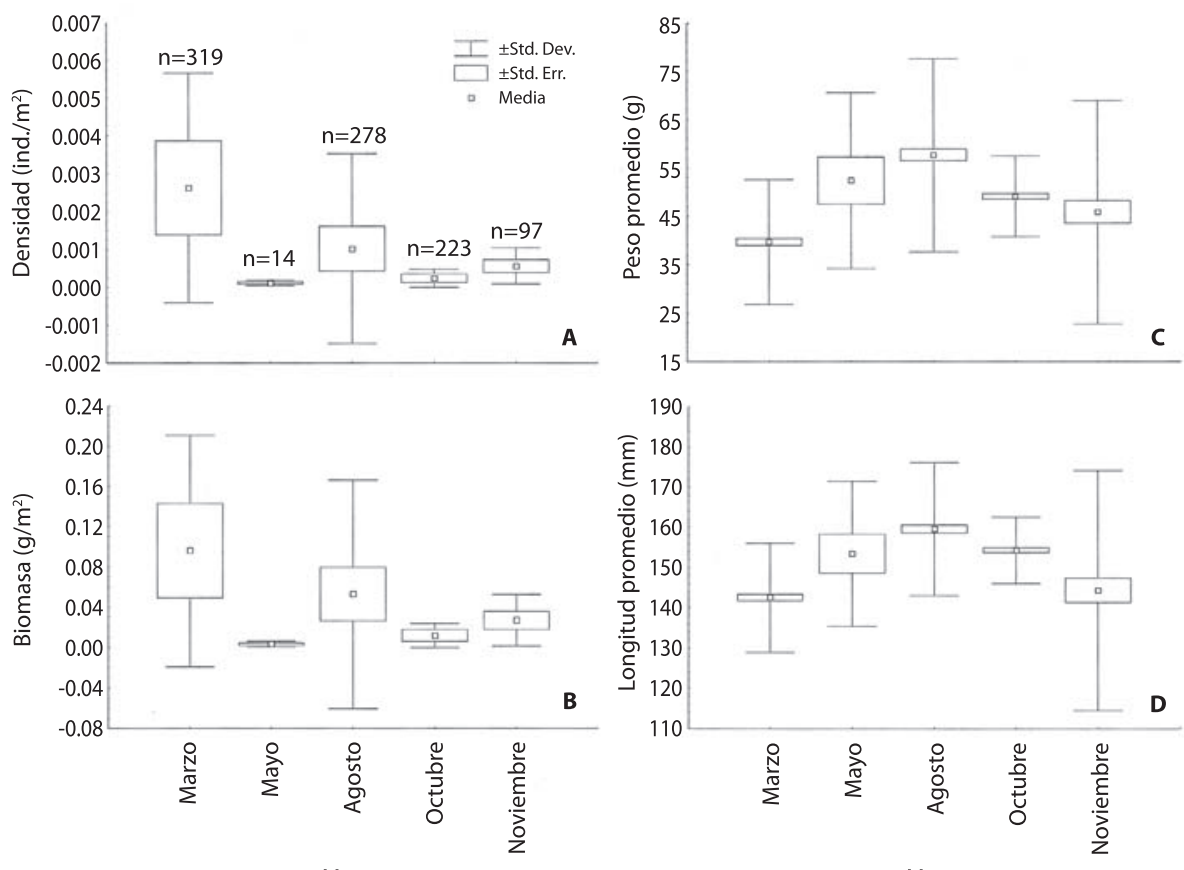

Meses

$$
\text { Meses }
$$

Fig. 3. Variación promedio de los parámetros poblacionales. A. Densidad. B. Biomasa. C. Peso. D. Longitud.

Fig. 3. Average variation of the population parameters. A. Density. B. Biomass. C. Weight. D. Length. 
marcado incremento progresivo durante los primeros meses analizados, alcanzando el valor máximo en agosto y octubre ( $\bar{x}=159.5 \mathrm{~mm}$ y $\bar{x}=57.65$ gr, respectivamente), y decrecen en los meses posteriores (Figs. 3c, 3d). La distribución espacial de la longitud muestra que los individuos de mayor talla se distribuyen al sureste del Golfo de Tehuantepec, cercanos a la isobata de los $60 \mathrm{~m}$, y los individuos de menor talla se distribuyen frente a los principales sistemas estuarinos de la zona (Laguna Superior-Inferior y Laguna del Mar Muerto). El análisis de frecuencia de tallas muestra una posible distribución modal para los meses de marzo, agosto y octubre, con una distribución bimodal y multimodal para los meses de mayo y noviembre respectivamente (Fig. 4).

Reproducción: El análisis de proporción sexual hembras:machos, para todos los meses analizados, presenta una relación cercana a 1:1, correspondiendo el $44 \%$ hembras, el $51 \%$ a machos y el $5 \%$ a indeterminados (jóvenes). Durante los meses analizados la proporción de machos es alta en marzo, mayo y agosto, disminuyendo en octubre y noviembre. Las hembras presentan un comportamiento inverso al de los machos, con la máxima proporción en octubre y noviembre y menor proporción para el resto de los meses. En estos últimos meses la proporción hembras:machos llega a ser cercana a 1:1, y es más evidente durante el mes de noviembre. Los individuos sexualmente indeterminados (jóvenes) representan una fracción pequeña (5 \%) del total analizado, con la mayor abundancia en marzo y noviembre (Fig. 5).

El índice gonadosomático presenta un patrón diferente en ambos sexos. Para los machos, el valor promedio máximo corresponde al mes de octubre $(\bar{x}=0.0158)$, con valores bajos para el resto de los meses. Para las hembras el valor promedio máximo se presenta durante el mes de mayo ( $\bar{x}=0.0284)$ con valores bajos para el resto de los meses analizados (Figs. 6A, B).

El índice visceral tiene, para las hembras, el valor promedio máximo en agosto $(\bar{x}=0.092)$ y el valor promedio mínimo en octubre $(\bar{x}=0.072)$. Los machos presentan el

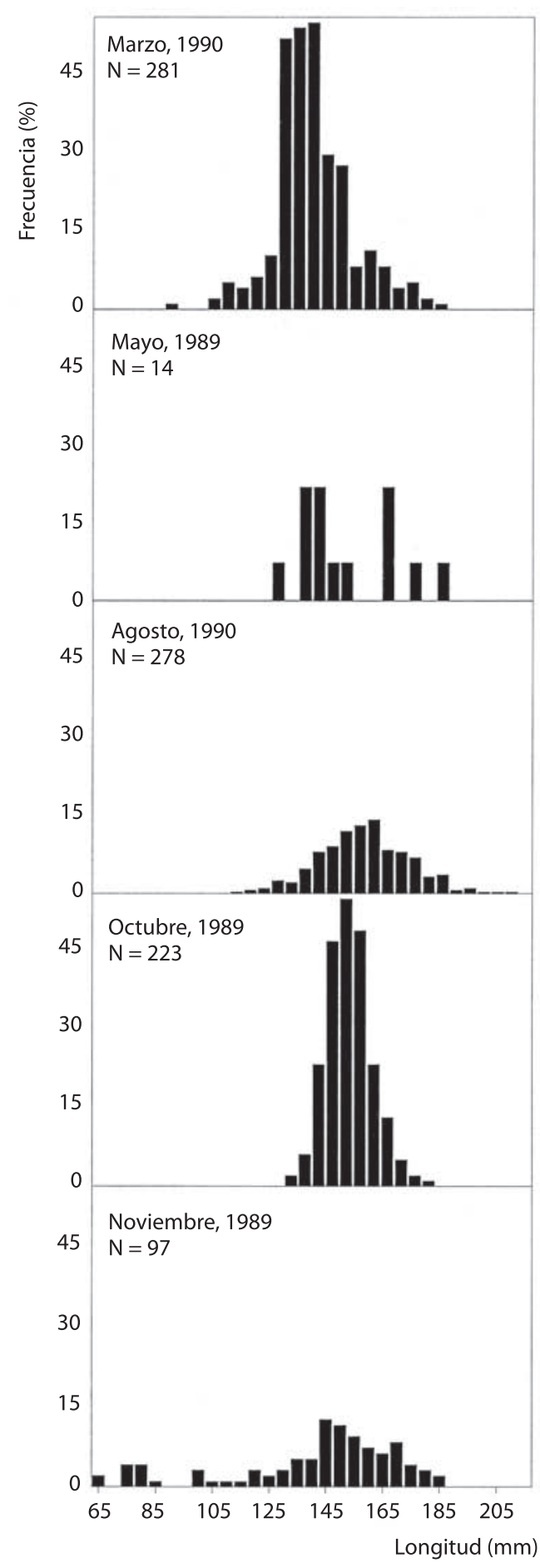

Fig. 4. Frecuencias de tallas de P. grandisquamis por mes. Fig. 4. Lenght frequencies of P. grandisquamis by month. 


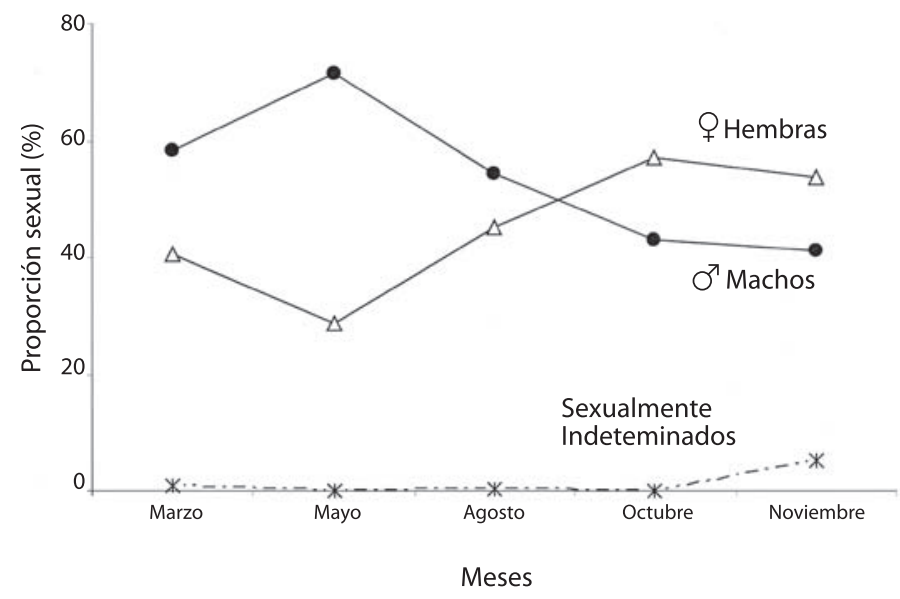

Fig. 5. Proporción sexual de P. grandisquamis.

Fig. 5. Sex ratio of P. grandisquamis.

valor promedio máximo en mayo $(\bar{x}=0.095)$ y el mínimo en marzo ( $\bar{x}=0.061$ ) (Figs. 6C, D).

Todos los estadios de madurez se presentan en todos los meses en diferente proporción; incluyendo los individuos sexualmente indeterminados (I). En octubre y mayo los organismos más pequeños (jóvenes) se encuentran sexualmente determinados (II), sin presentarse estadios en descanso (VII) (Fig.7).

La talla de primera madurez para $P$. grandisquamis fue de $138 \mathrm{~mm}$, debido a que la talla del individuo inmaduro más grande (II) fue de 161 mm de longitud total (noviembre), y el más pequeño en proceso de maduración (III) alcanzó una talla de $115 \mathrm{~mm}$ (noviembre) (Fig. 7). La distribución espacial de los individuos maduros muestra que se distribuyen a lo largo del Golfo de Tehuantepec, y se concentra la mayor parte frente a los sistemas lagunares de la región. Al

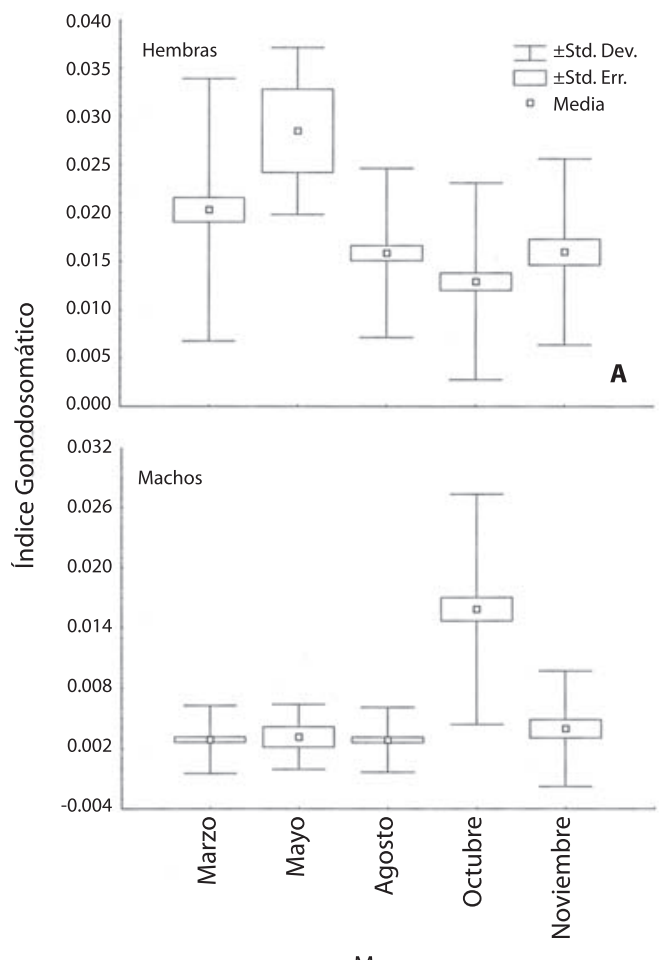

Meses

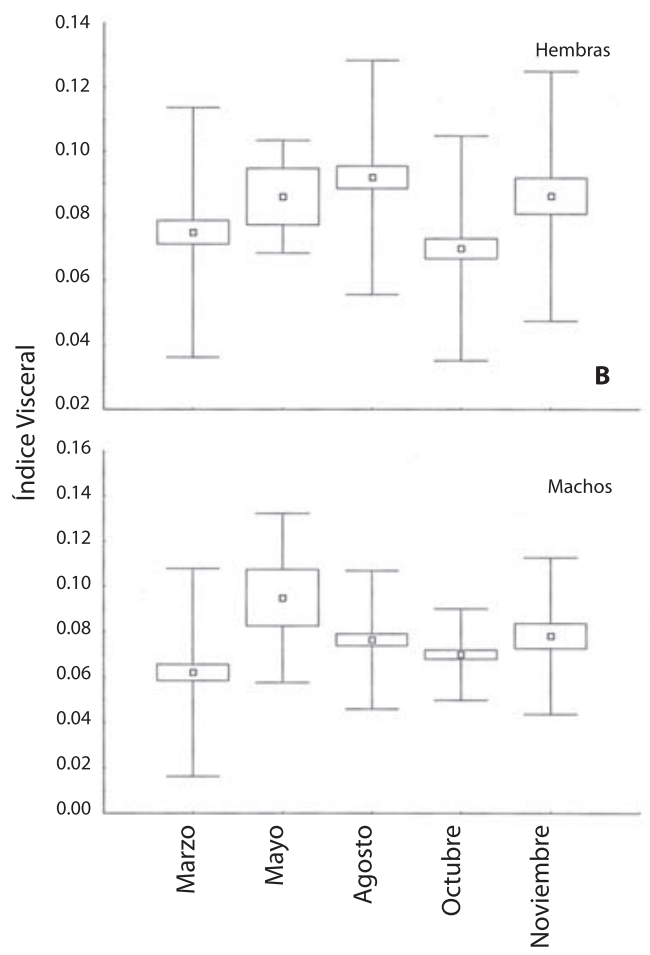

Meses

Fig. 6. Variación del índice visceral (A) y gonadosomático (B) para hembras y machos.

Fig. 6. Variation of the visceral (A) and gonadosomatic index (B) by sex. 


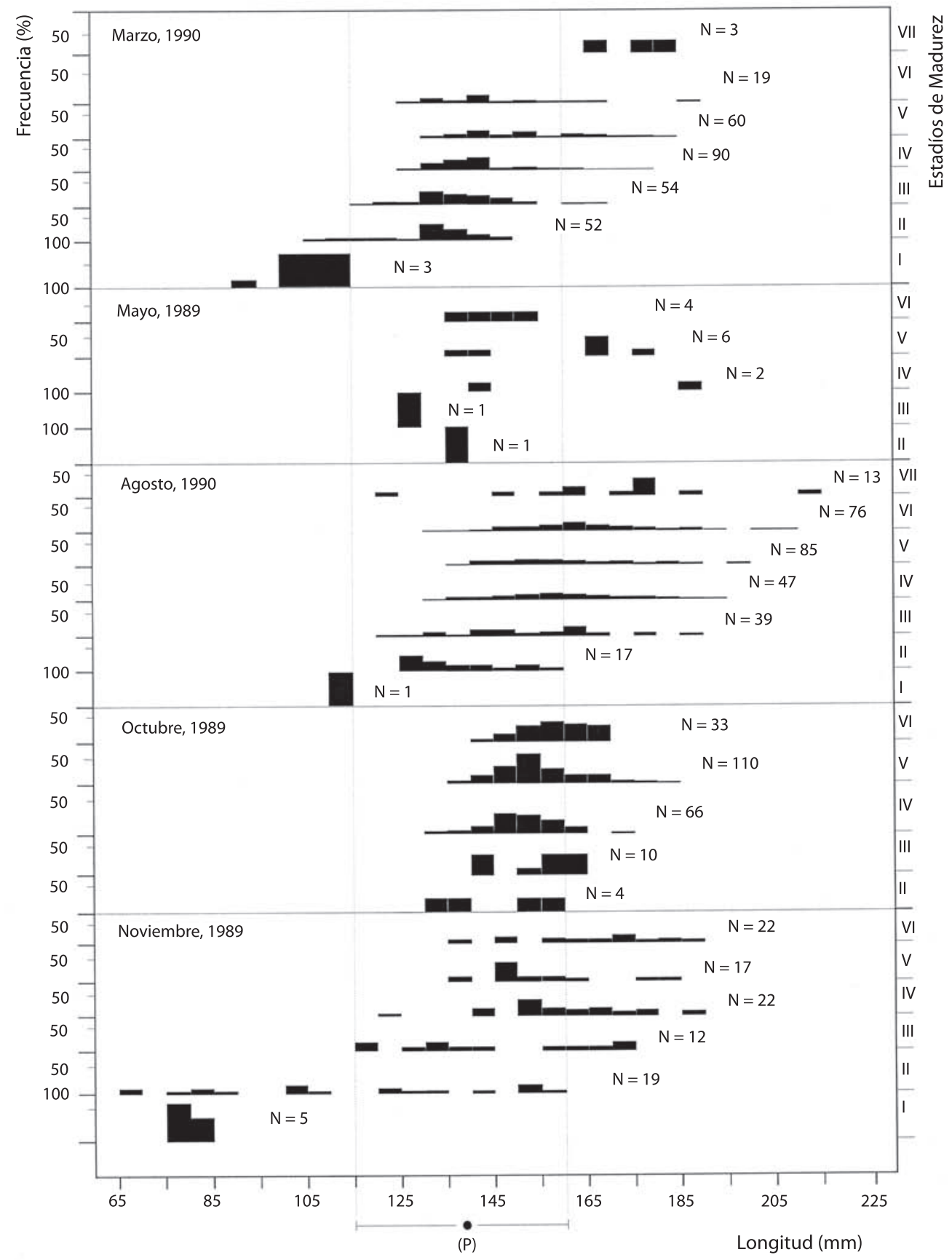

Fig. 7. Frecuencia de tallas de P. grandisquamis, y su relación con la madurez gonádica para cada mes de estudio. Talla de primera madurez $(\mathrm{P})$.

Fig. 7. Size frequencies of P. grandisquamis, and their relation to gonad maturity for each month studied. Size of first maturity (P). 
respecto, los individuos sexualmente indeterminados presentan una distribución asociada a procesos estuarinos, ocurriendo principalmente frente al sistema lagunar Superior-Inferior.

\section{DISCUSIÓN}

Distribución y abundancia: De acuerdo a Tapia-García (1998), P. grandisquamis es una de las especies consideradas como dominantes dentro del Golfo de Tehuantepec, y en conjunto con otras especies dominantes, aportan más del $80 \%$ de abundancia numérica en las capturas, durante los meses de marzo y agosto, con una frecuencia mayor al $45 \%$ durante los meses de marzo, agosto y noviembre.

La mayor abundancia alrededor de la isobata de los $40 \mathrm{~m}$, frente a los sistemas lagunares y hacia el sudeste del Golfo, sobre fondo arenoso y lodoso, hace evidente su relación a áreas influenciadas por sistemas lagunares-estuarinos, lo que concuerda con lo informado por Amezcua-Linares (1996) y Allen y Robertson (1998). Este patrón de relación a zonas de influencia por procesos estuarinos es una estrategia biológica y ecológica que permite a las especies aprovechar la alta producción de origen epicontinental. No existen informes de que esta especie se distribuya dentro de sistemas lagunares-estuarinos (Schneider 1995, TapiaGarcía 1997, Castro-Aguirre 1999), por lo que es una especie típica marina que se distribuye preferentemente hacia la plataforma continental interna. Este patrón es diferente al informado para Mulloidichthys dentatus, especie de la misma familia, que se reporta para el área de estudio y que se distribuye principalmente sobre fondos rocosos y arenosos, cerca de arrecifes coralinos (Allen y Robertson 1998).

La alta abundancia de $P$. grandisquamis en marzo está determinada principalmente por individuos de tallas juveniles, que se asocia a un fuerte reclutamiento. La alta abundancia en agosto se atribuye principalmente a individuos adultos de tallas más grandes respecto a marzo, lo que implica un crecimiento en los individuos de la población. La disminución de la biomasa en mayo y noviembre es resultado del escaso número de organismos recolectados y por la presencia de individuos de tallas pequeñas, principalmente durante el mes de noviembre, como resultado del reclutamiento de jóvenes.

La frecuencia de tallas muestra una progresión modal en los meses analizados, lo cual es un reflejo de un amplio proceso de crecimiento y reclutamiento de los organismos. La distribución multimodal durante los meses de noviembre a marzo muestra un amplio periodo de reproducción y reclutamiento asociado a la presencia constante de individuos sexualmente indeterminados (Fase I).

La talla máxima fue de $213 \mathrm{~mm}$, y al respecto Amezcua-Linares (1996) y Allen y Robertson (1998) informan una talla máxima de $250 \mathrm{~mm}$ y $280 \mathrm{~mm}$ respectivamente para el Pacífico Central Mexicano.

Reproducción: La proporción sexual total hembras:machos fue prácticamente 1:1 durante el ciclo anual. Sin embargo, en mayo donde la proporción es diferente respecto a los otros meses, puede deberse al bajo número de individuos recolectados. Por otro lado, en ambos sexos se presenta una diferenciación en cuanto a la madurez, lo cual puede indicar una posible separación de hábitat entre áreas reproductivas y no reproductivas, acercándose a la línea de costa para la reproducción y la alimentación.

La presencia de individuos indeterminados, principalmente en noviembre y marzo, sugiere un amplio periodo de reclutamiento. Esto se asume por la presencia de individuos en maduración (III), maduros (IV), en reproducción (V) y desovados (VI), lo que indica que la reproducción ocurre todo el año, principalmente durante el periodo comprendido en mayo a octubre, periodo que corresponde a la época de lluvias y que se caracteriza por una mayor disponibilidad de alimento y mejores condiciones ambientales que garantiza en gran medida el éxito de la sobrevivencia de los organismos. El periodo de desove más activo durante los meses de mayo a octubre, coincide con lo reportado por Wahbeh (1992) para Mulloides flavolineatus y $M$. vanicolensis en 
el Mar Rojo donde se informan desoves cortos estacionales de junio a agosto. El reclutamiento de jóvenes asociado a su alta abundancia frente a las Lagunas Inferior y del Mar Muerto permite inferir que estas áreas son de alimentación y crianza de los jóvenes de la especie y probablemente áreas reproductivas de los adultos, que permiten asegurar la continuidad de la especie por la producción que exportan los sistemas lagunares.

El análisis del índice gonadosomático también indica un amplio periodo reproductivo a partir de mayo hasta octubre. Esta conducta es característica de peces tropicales los cuales muestran un reclutamiento constante de jóvenes, ciclos de vida cortos y reproducción durante casi todo el año (Tapia-García et al. 2000). Por el número de modas que se presentan en la frecuencia de tallas es muy probable que $P$. grandisquamis tenga una longevidad mayor a tres años.

La talla de primera madurez sexual fue de $138 \mathrm{~mm}$ de longitud total, y tomando como referencia esta longitud, los individuos menores a esta talla tienden a distribuirse frente a las Lagunas Inferior y Mar Muerto, lo que corrobora a éstas como áreas de crianza.

Se puede resumir que esta especie típicamente marina se distribuye hasta $60 \mathrm{~m}$ de profundidad, donde los individuos de tallas mayores alcanzan las máximas profundidades para madurar. Posteriormente se dirigen hacia aguas someras frente a sistemas lagunares-estuarinos para reproducirse durante todo el año, principalmente en el periodo de mayo a octubre, periodo en el cual se registra la mayor producción de los sistemas lagunares. En estas zonas, por su alta producción constituyen áreas de crianza, donde se desarrollan los jóvenes hasta alcanzar su talla de primera madurez y se reclutan a la población adulta colonizando aguas de mayor profundidad, para completar su ciclo vital, principalmente en marzo y noviembre. La veda para la pesca de camarón en la región se aplica de mayo a septiembre, lo que determina que esta actividad no tenga un fuerte impacto durante el periodo reproductivo de la especie. De acuerdo con la información brindada por Tapia-García (1997,
1998) Tapia-García et al. (2000) y el presente trabajo, se sugiere aplicar estrategias de protección a las zonas sobre la plataforma continental del Golfo de Tehuantepec influenciadas por procesos estuarinos y que representan áreas de reproducción y crianza para un gran número de especies, para garantizar la permanencia de éstas en el sistema.

\section{AGRADECIMIENTOS}

El presente estudio deriva del apoyo obtenido a través del proyecto de investigación “Composición taxonómica, distribución y abundancia de las comunidades de peces de la zona costera de México: Bahías del Parque Nacional Huatulco. Universidad Autónoma Metropolitana-Iztapalapa/CBS”.

\section{RESUMEN}

Como resultado de sus estrategias biológicas y ecológicas, el pez Pseudupeneus grandisquamis es una especie dominante en la comunidad demersal del Golfo de Tehuantepec. Nuestro principal objetivo fue conocer estas estrategias con base en su distribución, abundancia y reproducción. Se analizaron un total de 5175 individuos que forman parte de las recolectas de peces obtenidas en cinco cruceros oceanográficos realizados en el Golfo de Tehuantepec, México, entre 1989-1990. Es una especie demersal típicamente marina, con amplia distribución en la plataforma continental. La mayor abundancia de $P$. grandisquamis se presenta en marzo y noviembre, alrededor de la isobata de los $40 \mathrm{~m}$ y frente a los sistemas lagunares Superior-Inferior y Laguna del Mar Muerto. La reproducción de la especie se presenta durante todos los meses analizados, principalmente durante los meses de agosto a octubre, correspondiente a la época de lluvias. La presencia de jóvenes, principalmente en noviembre y marzo, sugiere un amplio periodo de reclutamiento durante estos meses cuya distribución se refleja principalmente en el área influenciada por las lagunas Superior-Inferior, que representa un área de crianza. La proporción sexual total hembras:machos fue prácticamente 1:1. La talla máxima en la región es de $213 \mathrm{~mm}$ y la talla de primera madurez es de 138 mm LT. La alta abundancia y reproducción se presentan cuando el golfo tiene alta producción, acorde con la dinámica del sistema, donde tiene gran relevancia la influencia de las lagunas costeras. Se sugiere aplicar estrategias de protección a las zonas sobre la plataforma continental del Golfo de Tehuantepec influenciadas por 
procesos estuarinos y que representan áreas de reproducción y crianza para un gran número de especies, entre las que se encuentra $P$. grandisquamis.

Palabras clave: Pseudupeneus grandisquamis, reproducción, demersal, Tehuantepec, ecología, dominancia, lagunas.

\section{REFERENCIAS}

Allen, G.R. \& D.R. Robertson. 1998. Peces del Pacífico Oriental Tropical. Comisión Nacional para el Conocimiento y Uso de la Biodiversidad y Agrupación Sierra Madre, México, DF., México. 327 p.

Amezcua-Linares, F. 1996. Peces Demersales de la Plataforma Continental del Pacífico Central de México. Inst. de Cienc. del Mar y Limnología. UNAM, Comisión Nacional para el Conocimiento y Uso de la Biodiversidad. México, DF., México. 184 p.

Cailliet, G.M., M.S. Love \& A.W. Ebeling. 1986. Fishes: A field and laboratory manual on their structure, identification and natural study. Wadsworth, California, EEUU. 194 p.

Castro-Aguirre, J. 1999. Ictiofauna estuarino-lagunar y vicaria de México. Colección Textos Politécnico. Limusa, México, DF., México. 705 p.

Laevastu, T. 1971. Manual de métodos de Biología Pesquera. FAO. Acribia, Zaragoza, España. 243 p.

Pauly, D. 1982. Studying single - dynamics in a tropical multispecies context, p. 33-70. In D. Pauly \& G.I. Murphy (eds.). Theory and management of tropical fisheries. ICLARM Conference Proceedings 9. Manila, Filipinas.

Sánchez-Gil, P. \& A. Yáñez-Arancibia. 1985. Evaluación ecológica de recursos demersales costeros Tropicales; un enfoque metodológico en el Sur del Golfo de México, p. 175-314. In A. Yáñez-Arancibia (ed.). Recursos pesqueros potenciales de México: La pesca acompañante del camarón. Progr. Univ. de Alimentos.
Inst. Cienc. del Mar y Limnol., Inst. Nal. de pesca. UNAM, México, DF., México.

Schneider, M. 1995. Mullidae, p. 1299-1300. In W. Fischer, F. Krupp, W. Schneider, C. Sommer, K.E. Carpenter \& V.H Niem (eds.). Guía FAO para la identificación de especies para los fines de la pesca. Pacifico centro-oriental. Volumen II. Vertebrados-Parte I. FAO, Roma, Italia.

Stevenson, D.K. 1982. Una revisión de los recursos marinos de la región de la Comisión de Pesca para el Atlántico centro occidental (COPACO). FAO Doc. Tec. de Pesca. 211:1-146.

Tapia-García, M. 1997. Estructura e interacciones ecológicas de las comunidades de peces de la plataforma continental y la Laguna del Mar Muerto, en el Golfo de Tehuantepec al sur del Pacífico Mexicano. Tesis de Doctorado en Ciencias del Mar, Universidad Autónoma de México, México, D.F. 135 p.

Tapia-García, M. 1998. Evaluación ecológica de la fauna demersal, p. 129-148. In M. Tapia-García (ed.). El Golfo de Tehuantepec: el ecosistema y sus recursos. Universidad Autónoma Metropolitana-Iztapalapa, México, DF., México.

Tapia-García, M., A. Yáñez-Arancibia, P. Sánchez-Gil \& M.C. García-Abad. 1995. Distribución, abundancia y reproducción de Priacanthus arenatus Cuvier (Pisces: Priacanthidae), en la plataforma continental del sur del Golfo de México. Biotropica 27: 232-237.

Tapia-García, M., M.C. García-Abad \& G. Cerdenares Ladrón de Guevara. 2000. Reproduction, distribution and abundance of Bothus constellatus (Pisces: Bothidae), in the Gulf of Tehuantepec, México. Rev. Biol. Trop. 48: 205-213.

Wahbeh, M.I. 1992. Aspects of the reproduction biology and growth of two species of goatfish (Mullidae) from Aqaba, Red Sea. Senckenbergiana Maritima. Frankfurt/Main, Alemania. 22: 255-264.

Yáñez-Arancibia, A. \& P. Sánchez-Gil. 1988. Ecología de los recursos demersales marinos: fundamentos en costas tropicales. AGT. México, DF., México. 228 p. 